\title{
Research on Beauty Medical Health Monitoring System Based on Wireless Sensor Network
}

\author{
Xinwen Bi $\mathbb{D}^{1},{ }^{1}$ Shaocheng Song $\mathbb{D}^{1},{ }^{1}$ Haoyang Wang $\mathbb{D},{ }^{1}$ Yingjian Shao $\mathbb{D}{ }^{1}$ \\ Xiaodan Zhang $\mathbb{D},{ }^{2}$ Yanming Cheng $\mathbb{D}^{2},{ }^{2}$ and Ilkyoo Lee $\mathbb{B}^{3}$ \\ ${ }^{1}$ College of Computer Science and Technology, Beihua University, Jilin, China \\ ${ }^{2}$ College of Electrical and Information Engineering, Beihua University, Jilin, China \\ ${ }^{3}$ Division of Electrical, Electronics and Control Engineering, Kongju National University, Cheonan, Republic of Korea \\ Correspondence should be addressed to Xiaodan Zhang; zhangxd@beihua.edu.cn
}

Received 11 July 2021; Revised 27 August 2021; Accepted 8 September 2021; Published 24 September 2021

Academic Editor: Anand Nayyar

Copyright (c) 2021 Xinwen Bi et al. This is an open access article distributed under the Creative Commons Attribution License, which permits unrestricted use, distribution, and reproduction in any medium, provided the original work is properly cited.

Due to the gradual improvement of living standards in recent years and the continuous improvement of China's health care awareness, the aesthetic medicine model has gradually changed from the treatment mode of disease treatment to the prevention mode of early detection and early treatment. Meanwhile, wireless sensor networks (WSNs) are gradually infiltrating into the medical field due to their low power consumption, small size, wireless transmission, and portability which complies with the needs of the development of medical models. Therefore, this paper studies and implements a beauty medical health monitoring system based on WSN and ZigBee short-range wireless transmission protocol; moreover, wireless power transfer (WPT) technology is used for charging sensors. Experimental results demonstrate the feasibility that the maximum power levels converted in sensors by the WPT for different ISM bands can meet the average power consumption expected in the range of $200-500 \mu \mathrm{W}$. And the designed system can provide an important practical reference for promoting the informationization of telemedicine and the development of beauty medical systems in China.

\section{Introduction}

With the continuous deepening of the reform of the medical and health system, the Plastic Surgery Hospital of the Chinese Academy of Medical Sciences, in addition to solidly coordinating and implementing the 10 key tasks deployed by the National Health and Family Planning Commission, fully implements the budgetary final accounts, full cost accounting, and comprehensive performance appraisal management systems, exploring a reasonable salary system for medical staff and fully mobilizing the enthusiasm of the medical staff. The medical staff of the plastic surgery hospital thus have a dignified life [1-3]. It will further improve the normalization of the medical service action plan, continuously expand the outpatient appointment registration, minimize the waiting time for the patient's medical treatment process, increase the guidance service, improve the various forms of online payment methods, such as App, and improve the patient's satisfaction with the hospital service. Further, it will expand the medical association, establish cooperation with hospitals in other provinces, promote the professional skills of plastic surgery to grassroots medical and health institutions, and gradually form plastic surgery specialists through scientific research cooperation, specialist training, technical support, and remote consultation [4]. The plastic surgery profession has two major functions: birth defects and repair and functional reconstruction of traumatic tissue and organ defects, as well as medical cosmetic treatment [5-7]. Restoration and reconstruction therapy is the basic medical support function of public orthopaedic specialist hospitals and large public hospitals for plastic surgery; medical beauty services are for-profit medical projects. In the past 20 years, China's medical beauty industry has developed rapidly, but the beauty medical health monitoring system has lagged behind. 
According to the survey, patients with beauty and medical care are often unable to balance their careers and rehabilitation. It enables users to monitor their beauty and health in a more convenient and quick way through remote systems, solve problems in a timely manner through online doctorpatient consultation and communication systems to solve simple diseases, and reduce unnecessary links and troubles. Even if certain conditions are not resolved in time, you can find the lowest cost treatment plan as soon as possible and do work and family balance [8-11]. The emergence of remote aesthetic medical monitoring has fundamentally solved the above problems [12-14]. In general, remote health monitoring refers to the use of currently mature and widely used and stable communication technologies. Human physiological signals collected by the medical device at the detection end are packaged and sent to the system platform for comprehensive data processing and analysis. Then, through the Internet system platform, a method for providing diagnosis and treatment advice through instant communication is given [15]. It has evolved with the development of wireless interconnect technology, modern communication technology, and medical technology [16-19]. In this context, the rapid development of wireless Internet has brought new opportunities for telemedicine services, and the combination of the two has created new medical methods. This approach enables people to share and deliver medical information and data between patients and hospitals, between hospitals and hospitals through self-service detection and network interconnection and realize remote consultation, video medical conference, and other functions [20, 21]. Family-oriented telemedicine health monitoring refers to connecting a patient or monitored subject to a diagnosing doctor or hospital with advanced and appropriate medical equipment, models for telemedicine monitoring, diagnosis, treatment, rehabilitation, and care [22]. Remote patient monitoring is receiving increasing attention.

Based on the analysis above, this paper presents a beauty medical health monitoring network platform based on smart wireless sensors, which installs various vital signs monitoring nodes on the monitored human body. By using a WSN for data transfer, the vital signs of monitored people can be tracked via a computer or portable embedded handheld. Diagnosis and treatment are performed for the first time. In order to maintain the sufficient power of the sensors, WPT wireless charging mode is adopted. The sensor node installed on the monitored object can get rid of the constraint of the transmission line used by the traditional instrument, and the monitored person can move freely within a certain range, which does not affect his normal activities; therefore, it is more conducive to long-term realtime monitoring and providing patients with a more comfortable monitoring environment. Long-term real-time data can provide more information about the patient's condition diagnosis.

\section{Proposed Method}

2.1. Wireless Sensor Networks. The sensor network implements three functions of data acquisition, processing, and transmission. Together with communication technology and computer technology, it constitutes the three pillars of information technology. A WSN is a wireless network of a large number of fixed or mobile sensors that are cooperatively aware in a self-organizing and multihop manner. It collects, processes, and transmits information by sensing the geographic sensing area of the object. This information is ultimately sent to the network owner. WSNs have many types of sensors that detect various phenomena in the surrounding environment, including earthquakes, electromagnetics, temperature, humidity, noise, light intensity, pressure, soil composition, size, speed, and direction of moving objects. Potential application areas can be summarized as follows: military, aviation, explosion-proof, disaster relief, environment, medical, home, industrial, commercial, and other fields.

A WSN is a multihop self-organizing network system consisting of a large number of inexpensive microsensor nodes deployed in the surveillance area. The goal is to collaboratively perceive, collect, and process the perceived areas in the network coverage area [23]. Information about the object will be sent to the viewer. Sensors, sensing objects, and observers form the three elements of a WSN. The rapid development of MEMS, system-on-chip, wireless communication, and low-power embedded technology has spawned WSNs. Information awareness comes with low power, low cost, and distributed and self-organizing capabilities. On-site changes. A WSN is a multihop ad hoc network formed by a large number of inexpensive microsensor nodes deployed in a surveillance area and formed by wireless communication. Many people think that the importance of this technology is comparable to the Internet: just as the Internet enables computers to access a variety of digital information regardless of their storage location, sensor networks can extend the ability of people to interact remotely with the real world. It is even referred to as a completely new type of computer system because it differs from the ubiquitous features of previous hardware and the ability to collectively analyze. However, in many ways, when the Internet is connected to less than 200 universities and military laboratories, WSNs are like the original Internet, and researchers are experimenting with various communication protocols and addressing schemes. As shown in Figure 1, today, most sensor networks connect fewer than 100 nodes, and more nodes and communication lines make them very complex and difficult to use. Another reason is that the price of a single sensor node is currently not low and battery life can only last for a few months. But these problems are not insurmountable. WSNs have many types of sensors that detect various phenomena in the surrounding environment, including earthquakes, electromagnetics, temperature, humidity, noise, light intensity, pressure, soil composition, size, speed, and direction of moving objects. MEMS-based microsensor technology and wireless network technology provide broad application prospects for the WSN. These potential applications can be summarized as follows: military, aviation, antiterrorism, explosion protection, disaster relief, environmental, medical, home, industrial, commercial, and other fields. 


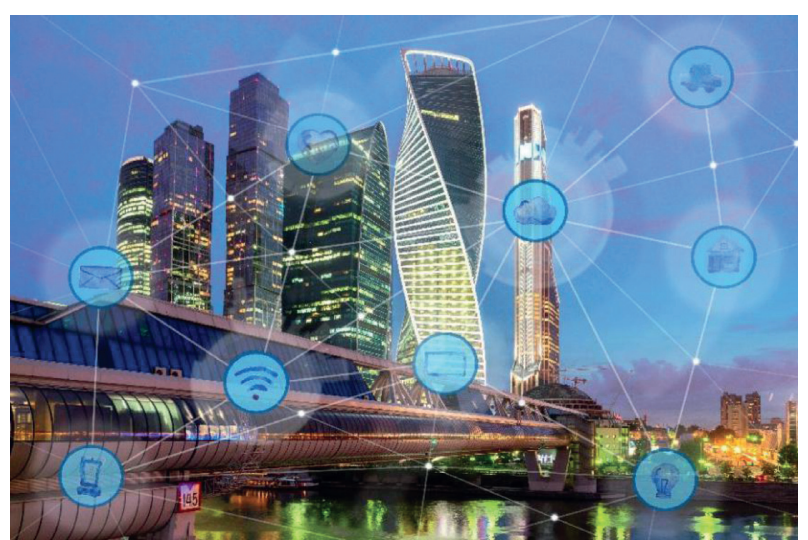

Figure 1: Sensing city (from the network https://www.cnblogs. com/hdawen/p/9572022.html).

Currently, most deployed WSNs are limited to collecting scalar data such as temperature, humidity, location, light intensity, pressure, and biochemistry. In practical applications such as medical monitoring, traffic monitoring, and smart home, we need to obtain multimedia information such as video, audio, and images. There is an urgent need for a new WSN, the wireless multimedia sensor network. The wireless multimedia sensor network is a new sensor network that introduces multimedia information sensing functions such as video, audio, and image on the basis of the traditional WSN. The wireless multimedia sensor network is a sensor node that collects richer video, audio, images, and other information in a WSN. These distributed nodes form a distributed sensor network with storage computing and communication capabilities. The WMSN senses various media information in the surrounding environment through the multimedia sensor node and can transmit information to the aggregation node through single-hop and multihop relay. The aggregation node then analyzes and processes the received data and finally sends the results of the analysis to the user [24]. This enables comprehensive and effective environmental monitoring.

2.2. Node Hardware and Software Design. The hardware design of the sensor node is divided into four functional modules: sensor module, processor module, wireless transceiver module, and power module as shown in Figure 1. The sensor module is designed with different sensors according to different acquisition signals. Basically, it covers the data needed for the initial diagnosis. The processor module uses TI's MSP430 family of 16-bit low-power processors with $60 \mathrm{kB}+256 \mathrm{~B}$ Flash, $2 \mathrm{kB}$ RAM, and a 6channel 12-bit ADC. The processor features ultra-low-power consumption, high processing speed, and rich interface. The wireless communication module selects Chipcon's CC2430 wireless transceiver module. The module features low voltage, low power, programmable output power, high sensitivity, small size, and integrated bit synchronizer. The main operating parameters can be programmed through the serial bus interface, very flexible. Because the chips used in power modules consume less power, they are powered by the most common $9 \mathrm{~V}$ alkaline batteries. The hardware structure of the sensor node is shown in Figure 2.

Node software design: the software portion of this node is based on the free downloadable ZigBee protocol stack (ZStack) from Texas Instruments. It complies with the ZigBee 2006 specification and supports multiple platforms, including the CC2430 system-on-chip solution for IEEE802.15.4/ZigBee, a new platform based on the CC2420 transceiver and the TIMSP430 ultra-low-power MCU. The protocol stack consists of the physical layer, media access control layer, network layer, security layer, and advanced application specifications. The security layer mainly implements functions such as key management and access. The application interface is responsible for providing users with a simple application software interface (API), including application subsupport (APS) and ZigBee device object (ZDO), to implement application layer-to-device management.

The node software design is based on the protocol stack and uses its rich functional modules in the component library to build its own APP functionality. The software development tools for the nodes in the platform use IAR Embedded Workhench, a compiler designed for the MSP430 microcontroller. Sensor node programming differs depending on the sensor it is equipped with. The basic programs involved in this platform are $\mathrm{A} / \mathrm{D}$ conversion, serial port transmission, and timing counting. The overall design flow of the program is shown in Figure 3.

The coordinator node program is mainly used to set parameters for establishing and starting network processes to achieve point-to-point connections. Therefore, no sensor modules are required. Through the serial port to communicate with the host, the overall design flow of the program is as follows: with initialization after power on, finding the binding request waiting for the router node, and assigning a short 16-bit address to each node in the network, the network succeeds; waiting for the host command, then the host command is sent to the router in the network through broadcast; and after receiving the data sent by the router, the data is sent to the host through the serial port. The program flowchart is shown in Figure 4.

\subsection{Beauty Medical Electronic Monitoring System Based on} Wireless Sensor Network. WSNs collect information by arranging a large number of wireless nodes and are considered one of the top ten technologies to change the world because of its convenient networking, flexible configuration, simple nodes, and low cost. It has great application prospects in solving patients' timely and effective monitoring. An electronic health monitoring system constructed using WSN has the following advantages: (1) it is a multiparameter measurement requirement that satisfies a patient's vital signs; (2) it changes the monitoring equipment from wired to wireless, reduces the patient's psychological pressure, and improves the accuracy of the data; (3) using the wireless network, the monitoring information can be sent to the doctor in a timely and effective manner; (4) because the cost of WSN nodes and networks is very low, the economic 


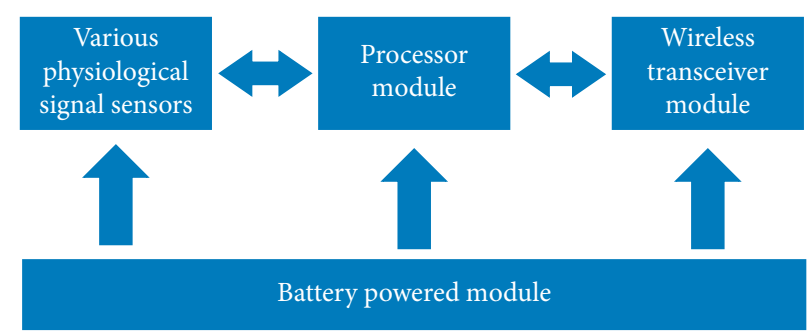

FIgURE 2: Sensor node hardware block diagram.

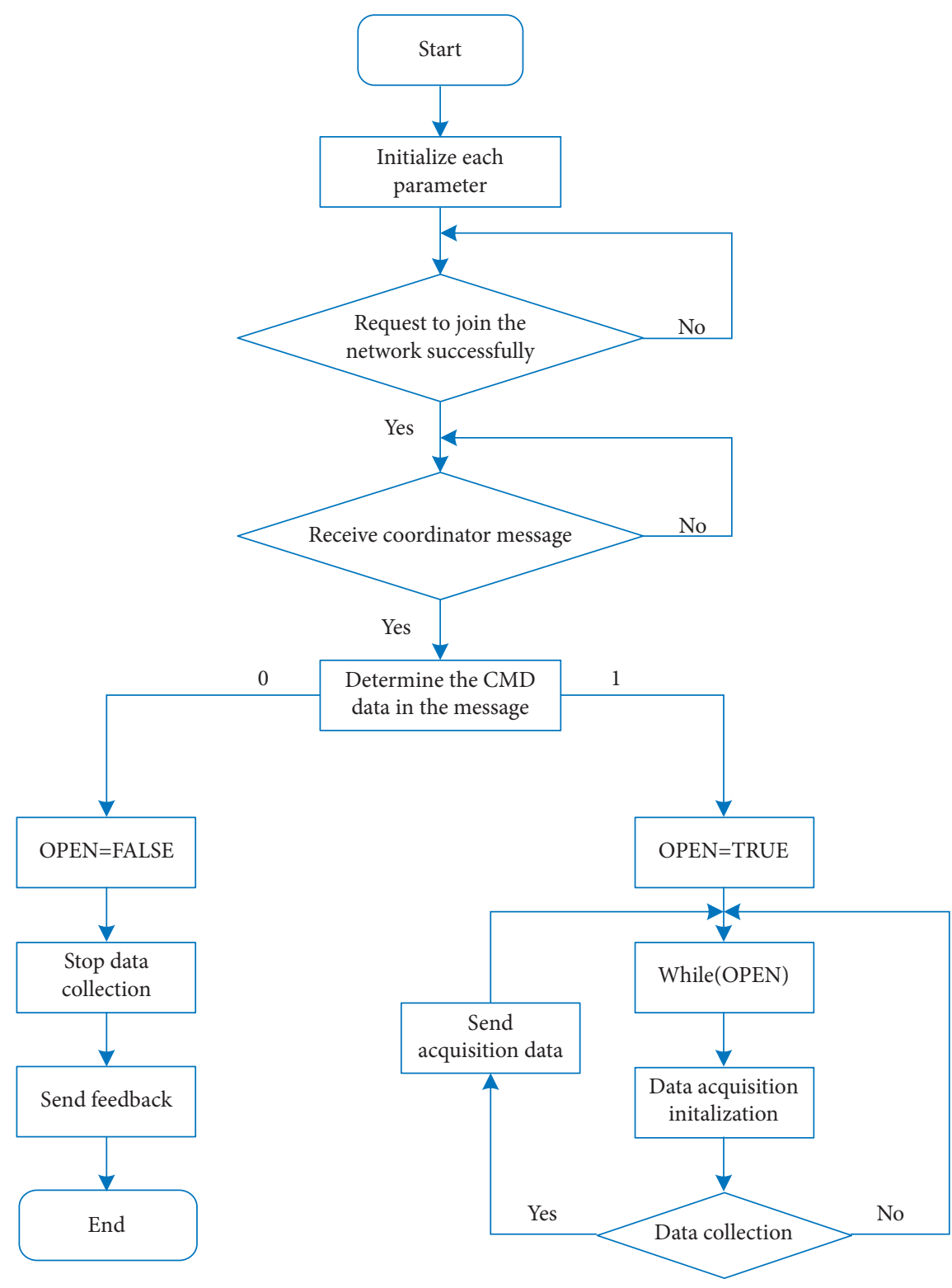

FIgURE 3: Sensor node program flowchart.

burden on patients is greatly reduced; and (5) it can be an important part of hospital informationization.

The design of the WSN subsystem node software is done within the new operating system TinyOS. TinyOS is an embedded operating system for sensor networks. Concurrency-intensive operations that support network sensors with minimal hardware are implemented. The node software design mainly includes layered routing protocols based on WSNs and software module development of WSN nodes. The routing protocol is responsible for forwarding data from the source node to the destination node over the network; finding the optimized path between the source node and the 


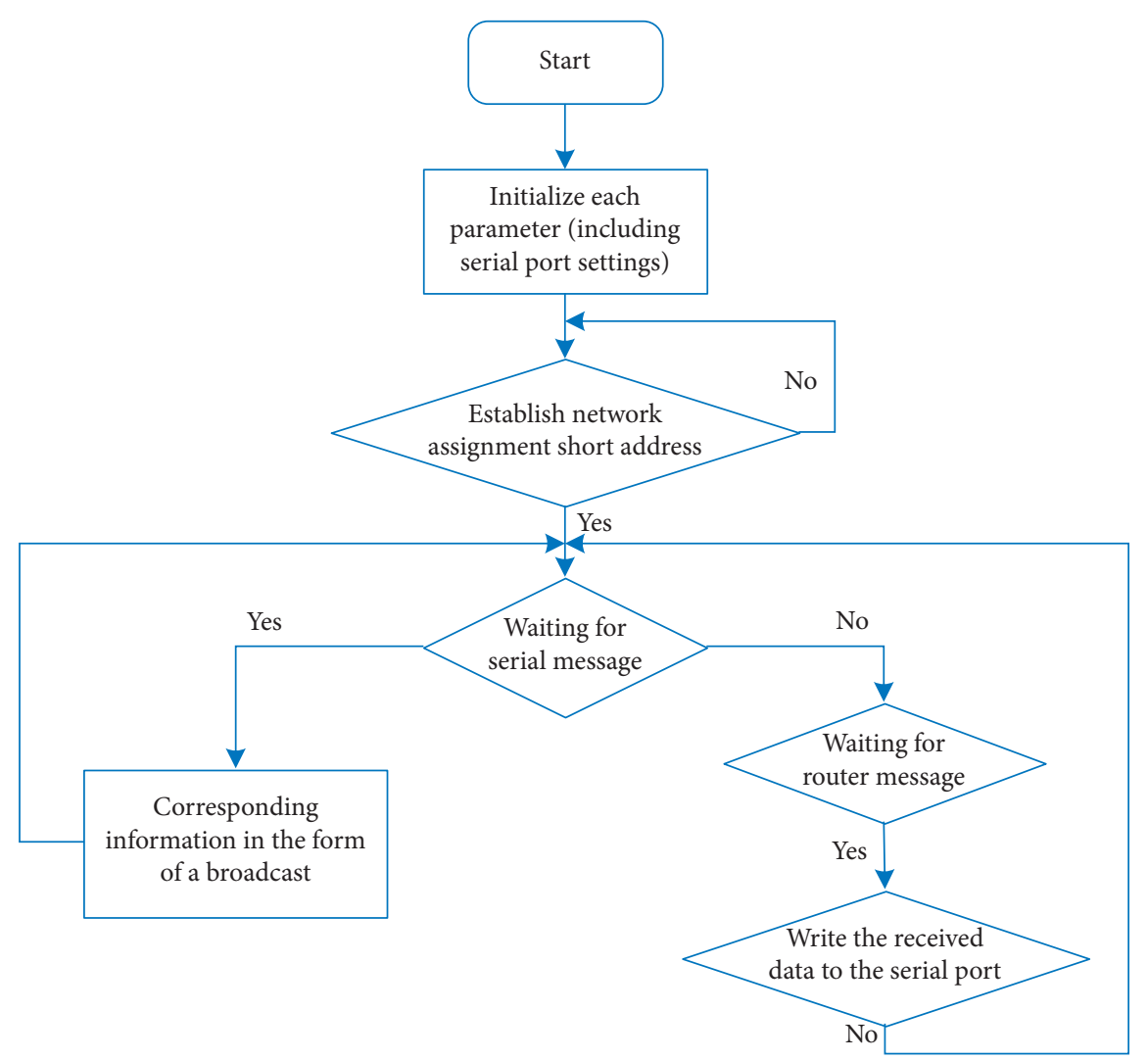

FIgURE 4: Coordinator node program flowchart.

target node; and forwarding the packet correctly along the optimized path. According to the resulting topology, WSN routing protocols can be divided into planar routing protocols and hierarchical routing protocols. In a planar routing protocol, all nodes have the same state and there is no bottleneck in principle. The disadvantage is poor scalability, and maintaining a dynamically changing route requires a lot of control information. In a hierarchical network, the function of group members is relatively simple and does not require maintenance complex routing information, which greatly reduces the number of network routing control information. It has good scalability, and its disadvantage is that the group head node may become the bottleneck of the network [25]. Based on the characteristics of the system, a hierarchical routing protocol based on WSN is designed. Taking into account the characteristics of the structure monitoring WSN applications in a software development process. This paper has made corresponding improvements in the following three aspects to ensure the normal operation of the network while saving energy as much as possible: the collecting node receives the acquisition command of the relay node to perform the function of collecting experimental data; otherwise, it is in a sleep state to save energy; transmission state under the node energy consumption is much larger than the energy consumption in the other states, and the software from the experimental data collected in the data processing and transmission, reducing the flow rate, saves energy; and the energy consumption is proportional to the square of the distance. The wireless sensor network subsystem can implement its data acquisition, forwarding, and receiving functions. The relay node starts transmitting the flag start signal, and after the acquisition node receives the flag start signal, the timer triggers to start collecting data. At the same time, based on its own node number, it finds its own cluster and sends the collected and processed data to the cluster head of the cluster. It is then forwarded by the cluster head to the relay node and finally sent to the user via serial communication.

Different application scenarios require different solutions. For example, in homes and hospitals, the solutions used are definitely different due to the long distances. The WSN + WLAN solution adopted by the hospital is more suitable. The transmission of various services after the network architecture is SMS, e-mail, or real-time parameter transmission and must also be combined with the actual needs of doctors and patients. The electronic monitoring system based on WSN mainly solves two technical problems. Key technologies for WSN: WSNs integrate multiple technologies such as sensors, embedded computing, modern networks and wireless communications, and distributed systems. It is a current research hotspot and has achieved many research results, but there are still many key technologies that need to be broken. The key technologies of WSN can be divided into three categories: application layer key technologies: including synchronization technology, positioning technology, middleware technology, simulation technology, application layer software, and industry solutions. Hierarchical protocol stack: the wireless layer 
technology includes a physical layer, link layer MAC protocol, network layer routing protocol, and transport layer transmission control; management technology includes energy management, topology control, QoS, security, network management, and mobility management. Biomedical signal processing technology: traditional biomedical signal information processing methods are based on Fourier analysis theory. Combined with the research tasks of this project, our main research objectives will be applied to medical signal processing such as wavelet transform, adaptive filtering, and independent component analysis, the ultimate solution for achieving system signal acquisition and processing goals [26].

\section{Experimental Results and Analysis}

The PC is the control center of the entire network, communicates with the coordinator through the serial port, controls the data transmission and reception in the wireless network, and acquires the signal of the sensor node. It is also an interactive platform of the human-machine interface through which the guardian queries the vital signs of the ward. The program includes serial communication module, data acquisition real-time drawing module, network control module, and data storage analysis module. The program development tool is Microsoft Visual Studio 2017, which is the current popular Windows platform application development environment for creating Windows applications and web applications on the Windows platform. It can also be used to create web services, smart device applications, and Office plugins. A smart device application based on the wince 5.0 operating system running on ARM9 can be developed by installing the corresponding SDK. The controls used throughout the application development process are common controls for dialogs, which are commonly used by ARM9. Therefore, it is very convenient to transplant in the future. The data analysis module uses the most basic ECG QRS waveform recognition algorithm. The overall flow of the program is as follows: first, to accurately identify the waveform must eliminate noise interference, so it is necessary to filter the transmitted raw data. The program uses a five-point smoothing filter function to make the processed data fall on the smooth curve, reducing statistical errors and improving measurement accuracy. Signal conditioning circuit designed to accommodate multichannel signal conditioning strain and used in conjunction with a WSN having 8 nodes ADC channels. This allows a wider area to be monitored with less hardware. This portion includes an amplifying circuit and a second-order low-pass filter circuit. The main components are the single-supply linear amplifier AD623 and the operational amplifier OPA340. How it works: connect the strain gage to the half-bridge circuit, convert the microstrain signal into an electrical signal, and adjust the potentiometer $\mathrm{Rg}$ to change the amplification gain of the AD623 to amplify the signal. It is then filtered by a second-order filter circuit and finally sent to the wireless sensor node for processing.

The WSN coverage control plays an important role in WSNs. To alleviate unnecessary energy wastage and improve network performance, we consider both energy efficiency and coverage rate for WSNs [27, 28]. To analyze whether the target application is feasible, we first briefly analyze the energy utility of the application. The most advanced accelerometer (e.g., LIS2DE12TR from ST Microelectronics or Bosch Sensortec) consumed an average of about 30-300 $\mu \mathrm{W}$ at the event, depending on the sampling rate. For the temperature sensor (e.g., from Silicon Labs, Austin, Texas, USA SI7053-A20-IM), the average power per second (sps) measured is less than $10 \mu \mathrm{W}$. The power consumption of the system-on-chip that forms the BLE transceiver and microcontroller is less than 30 when the microcontroller is active and idle. In the case of microwatts, the transmission is approximately 60 milliwatts. Therefore, the average power consumption is expected to be in the range of $200-500 \mu \mathrm{W}$. The specifications of WPT in different subbands are summarized in Table 1.

Both the WPT transceiver and receiver antennas are omnidirectional (e.g., a dipole with a gain of $2.15 \mathrm{dBi}$ ) and the efficiency of the RF-DC conversion is $50 \%$. In addition, we use a free-space propagation model to account for losses in the channel. Consider RF energy transmitted in the $2.4 \mathrm{GHz}$ band with a maximum effective isotropic radiated power (EIRP) of $10 \mathrm{~mW}$. At a distance of one meter, the propagation loss is approximately $37 \mathrm{~dB}$ and the total acquisition power is approximately $100 \mu \mathrm{W}$, which is lower than the level required for our target application. Next, we consider the h1.6 band with $500 \mathrm{~mW}$ effective radiated power and a $10 \%$ duty cycle limit. At a distance of about 2.8 meters, the harvested power exceeds $0.5 \mathrm{~mW}$, so that the target device can be supplied. Finally, for the band g3, it can be estimated that a target level of $500 \mu \mathrm{W}$ is satisfied at a distance of about 2.5 meters. Figure 5 summarizes the computable maximum power levels converted by the WPT for different ISM bands, taking into account the duty cycle limits.

The most important transmission module detection module for mobile devices facing important issues is flexibility. The flexibility here mainly refers to the interruption of data transmission caused by the system or device using interrupt power or due to the instability of the network environment. The flexibility that the system needs is the processing scheme for data saving and recovery transmission in this case. This paper considers this issue when designing system performance. Next, waiting for the transfer command, such a design is beneficial to the data retransmission problem when the system occurs under the above circumstances, but at the same time, there is a problem that the storage capacity of the entire system is higher. Because in this system, the size of each complete measurement data does not exceed $1 \mathrm{kB}$, the contradiction between improving flexibility and improving system hardware requirements is not obvious. However, this is a large system in a very important factor, it cannot be ignored. It can be seen from the reliability analysis part of the system that when the time is long enough, the stability and timeliness of the system reach a steady state. That is to say, the packet loss phenomenon is still very obvious at this time, but as the delay of packet loss retransmission 
TABLE 1: Subband of non-specific short-range devices.

\begin{tabular}{lccc}
\hline Subband (ERC 70-03) & Frequency $(\mathrm{MHz})$ & Effective radiated power $(\mathrm{mW} / \mathrm{dBm})$ & Duty cycle $(\%)$ \\
\hline g1 & $433.050-434.790$ & $\leq 25 / 14$ & $\leq 10$ \\
g2 & $433.050-434.790$ & $\leq 1 / 0(-13 \mathrm{dBm} / 10 \mathrm{kHz})$ & No restriction \\
g3 & $433.040-434.790$ & $\leq 10 / 10$ & No restriction \\
h1.1-1.3 & $863.000-870.000$ & $\leq 25 / 14$ & $\leq 0.1$ \\
h1.4 & $868.000-868.600$ & $\leq 25 / 14$ & $\leq 1$ \\
h1.5 & $868.700-869.200$ & $\leq 25 / 14$ & $\leq 0.1$ \\
h1.6 & $869.400-869.650$ & $\leq 500 / 27$ & $\leq 10$ \\
h1.7 & $869.700-870.000$ & $\leq 5 / 7$ & No restriction \\
h1.7 & $869.700-870.000$ & $\leq 25 / 14$ & $\leq 1$ \\
h2 & $870.700-873.000$ & $\leq 25 / 14$ & $\leq 1$ \\
i & $2400.000-483.500$ & $\leq 10 / 10($ EIRP) & No restriction \\
j & $5725.000-5875.000$ & $\leq 25 / 14($ EIRP) & No restriction \\
\hline
\end{tabular}

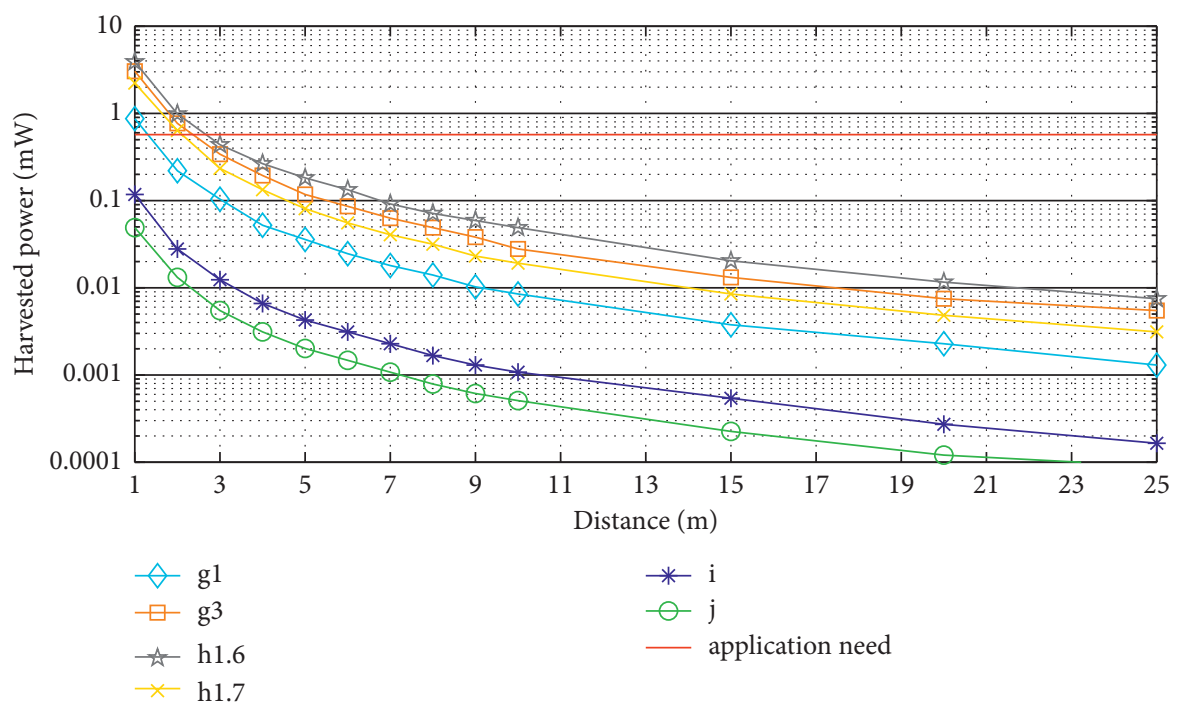

FIgURE 5: Comparison of the maximum power of the medical band transmitted by wireless power transmission.

increases, the data corruption situation is improved and eventually stabilized. At this point, the system works with maximum delay; therefore, a way should be researched to make the system transmit data as small as possible. The short-latency working method is also the key problem to be solved in the next step of the system design.

\section{Conclusions}

The patient's information can be presented to the medical staff better and faster, and the medical staff can also take ambulance measures more effectively, so as to better protect the patient's health recovery. At present, WSN technology is still developing rapidly, speeding up the research of wearable WSN technology, and gradually solving current difficulties and challenges. In the future, more comprehensive and efficient wearable WSN technology will be applied in the field of medical rescue, with unparalleled practical application value. Therefore, this paper designs a beauty medical health monitoring system based on WSN and ZigBee transmission protocol. Moreover, WPT technology is applied to charge sensors of WSN. Experimental results verified the feasibility and the flexibility of the system. In addition, the designed system has important practical significance for promoting the informationization of telemedicine and the development of beauty medical systems in China.

\section{Data Availability}

The data used to support the findings of this study are available from the corresponding author upon request.

\section{Conflicts of Interest}

The authors declare that there are no conflicts of interest regarding the publication of this paper.

\section{Acknowledgments}

This work was supported by the Education Department of Jilin Province (Grant JJKH20210047 KJ), Beihua University research project (Grant 202003015), 2019 Federation of Social Sciences Project of Jilin Pity (Grant 2199), and the National Social Science Fund Projects (Grant 19BTQ057). 


\section{References}

[1] S. Xie and Y. Wang, "Construction of tree network with limited delivery latency in homogeneous wireless sensor networks," Wireless Personal Communications, vol. 78, no. 1, pp. 231-246, 2014.

[2] I. Khan, F. Belqasmi, R. Glitho, N. Crespi, M. Morrow, and P. Polakos, "Wireless sensor network virtualization: a survey," IEEE Communications Surveys \& Tutorials, vol. 18, no. 1, pp. 553-576, 2017.

[3] C.-F. Wang, J.-D. Shih, B.-H. Pan, and T.-Y. Wu, "A network lifetime enhancement method for sink relocation and its analysis in wireless sensor networks," IEEE Sensors Journal, vol. 14, no. 6, pp. 1932-1943, 2014.

[4] S. Ferdoush and X. Li, "Wireless sensor network system design using raspberry pi and arduino for environmental monitoring applications," Procedia Computer Science, vol. 34, no. 3-4, pp. 103-110, 2014.

[5] B. Prabhu, R. Mahalakshmi, S. Nithya, P. D. Manivannan, and S. Sophia, A Review of Energy Efficient Clustering Algorithm for Connecting Wireless Sensor Network Fields, Social Science Electronic Publishing, Rochester, NY, USA, 2017.

[6] G. Tuna, V. C. Gungor, and K. Gulez, "An autonomous wireless sensor network deployment system using mobile robots for human existence detection in case of disasters," Ad Hoc Networks, vol. 13, no. 1, pp. 54-68, 2014.

[7] X. Xiao, G. J. Wang, and M. A. Zheng, "Data fusion in wireless sensor networks using BP neural network," Technological Development of Enterprise, vol. 2014, no. 3, 2 pages, 2014.

[8] M. Ndiaye, G. Hancke, and A. Abu-Mahfouz, "Software defined networking for improved wireless sensor network management: a survey," Sensors, vol. 17, no. 5, p. 1031, 2017.

[9] H. Zhao, W. Jie, Z. Jie, L. Liu, and K. Tian, "A general selforganized tree-based energy-balance routing protocol for wireless sensor network," IEEE Transactions on Nuclear Science, vol. 61, no. 2, pp. 732-740, 2014.

[10] L. M. Borges, F. J. Velez, and A. S. Lebres, "Survey on the characterization and classification of wireless sensor network applications," IEEE Communications Surveys \& Tutorials, vol. 16, no. 4, pp. 1860-1890, 2014.

[11] C. C. Lai, C. K. Ting, and R. S. Ko, "An effective genetic algorithm to improve wireless sensor network lifetime for large-scale surveillance applications," in Proceedings of the IEEE Congress on Evolutionary Computation, pp. 22-30, Singapore, 2007.

[12] J. Rui, L. Xin, B. Yan et al., "A nested ecohydrological wireless sensor network for capturing the surface heterogeneity in the midstream areas of the Heihe river basin, China," IEEE Geoscience and Remote Sensing Letters, vol. 11, no. 11, pp. 2015-2019, 2014.

[13] M. Magno, T. Polonelli, L. Benini, and E. Popovici, "A low cost, highly scalable wireless sensor network solution to achieve smart LED light control for green buildings," IEEE Sensors Journal, vol. 15, no. 5, pp. 2963-2973, 2015.

[14] C. Zhan, Y. Zeng, and R. Zhang, "Energy-efficient data collection in UAV enabled wireless sensor network," IEEE Wireless Communication Letters, p. 1, 2017.

[15] M. S. Farash, M. Turkanović, S. Kumari, and M. Hölbl, "An efficient user authentication and key agreement scheme for heterogeneous wireless sensor network tailored for the internet of things environment," Ad Hoc Networks, vol. 36, no. P1, pp. 152-176, 2016.

[16] I. Khan, F. Belqasmi, R. Glitho, N. Crespi, M. Morrow, and P. Polakos, "Wireless sensor network virtualization: early architecture and research perspectives," Network IEEE, vol. 29, no. 3, pp. 104-112, 2016.

[17] A. Thakkar and K. Kotecha, "Cluster head election for energy and delay constraint applications of wireless sensor network," IEEE Sensors Journal, vol. 14, no. 8, pp. 2658-2664, 2014.

[18] G. S. Sara and D. Sridharan, "Routing in mobile wireless sensor network: a survey," Telecommunication Systems, vol. 57, no. 1, pp. 51-79, 2014.

[19] S. Lee, M. Younis, and M. Lee, "Connectivity restoration in a partitioned wireless sensor network with assured fault tolerance," Ad Hoc Networks, vol. 24, pp. 1-19, 2015.

[20] C. Peng, K. Qian, and C. Wang, "Design and application of a VOC-monitoring system based on a ZigBee wireless sensor network," IEEE Sensors Journal, vol. 15, no. 4, pp. 2255-2268, 2015.

[21] J. Yang, H. Zhang, Y. Ling, C. Pan, and W. Sun, "Task allocation for wireless sensor network using modified binary particle swarm optimization," IEEE Sensors Journal, vol. 14, no. 3, pp. 882-892, 2014.

[22] V. K. Chaurasiya, N. Jain, and G. C. Nandi, "A novel distance estimation approach for 3D localization in wireless sensor network using multi dimensional scaling," Information Fusion, vol. 15, no. 1, pp. 5-18, 2014.

[23] J. Wang, Y. Gao, C. Zhou, R. Simon Sherratt, and L. Wang, "Optimal coverage multi-path scheduling scheme with multiple mobile sinks for WSNs," Computers, Materials \& Continua, vol. 62, no. 2, pp. 695-711, 2020.

[24] J. Wang, Y. Gao, X. Yin, F. Li, and H.-J. Kim, "An enhanced PEGASIS algorithm with mobile sink support for wireless sensor networks," Wireless Communications and Mobile Computing, vol. 2018, Article ID 9472075, 2018.

[25] K. Vijayalakshmi and P. Anandan, "Global levy flight of cuckoo search with particle swarm optimization for effective cluster head selection in wireless sensor network," Intelligent Automation \& Soft Computing, vol. 26, no. 2, pp. 303-311, 2020.

[26] A. A. Hady, "Duty cycling centralized hierarchical routing protocol with content analysis duty cycling mechanism for wireless sensor networks," Computer Systems Science and Engineering, vol. 35, no. 5, pp. 347-355, 2020.

[27] J. Wang, C. Ju, Y. Gao, A. K. Sangaiah, and G. J. Kim, “A PSO based energy efficient coverage control algorithm for wireless sensor networks," Computers, Materials \& Continua, vol. 56, no. 3, pp. 433-446, 2018.

[28] S. ShayestehTabatabaei, "A novel fault tolerance energy-aware clustering method via social spider optimization (SSO) and fuzzy logic and mobile sink in wireless sensor networks (WSNs)," Computer Systems Science and Engineering, vol. 35, no. 6, pp. 477-494, 2020. 